\title{
APLICAÇÃO DO MÉTODO DE SALESKY ET AL. (2012) USANDO UM FILTRO TEMPORAL PARA ESTIMAR O ERRO DO FLUXO DE CALOR SENSÍVEL EM TIJUCAS DO SUL - PR E EM MISSAL - PR
}

\author{
Bianca Luhm Crivellaro $^{1}$, Nelson Luís Dias ${ }^{1,2}$ \\ ${ }^{1}$ Programa de Pós-Graduação em Engenharia Ambiental, UFPR; \\ ${ }^{2}$ Departamento de Engenharia Ambiental, UFPR;
}

\section{RESUMO}

O Método de Covariâncias Turbulentas é o mais usado para o cálculo de fluxos turbulentos; contudo, esse método pode possuir contribuições de vários tipos de erros. Levando em consideração o erro aleatório, Salesky et al. (2012) desenvolveram um método de filtragem para calcular o erro aleatório de momentos turbulentos de qualquer ordem. Neste trabalho, aplicamos este método, usando um filtro temporal para calcular o erro do fluxo de calor sensível. $\mathrm{O}$ valor médio encontrado para o erro foi de aproximadamente $7 \%$.

\section{ABSTRACT}

The eddy covariance method is the most used approach to calculate turbulent fluxes; however, this method can include contributions from several types of errors. Considering the random errors, Salesky et al. (2012) developed a filtering method to estimate the random error of turbulent moments of any order. In this paper, we apply this method, using a temporal filter, to calculate the error of the sensible heat flux. The mean value of the error among all runs analyzed was approximately $7 \%$.

\section{INTRODUÇÃO}

A ferramenta mais utilizada para a medição de fluxos turbulentos é o Método de Covariâncias Turbulentas (MCT). Esse método consiste em medições simultâneas em alta frequência da velocidade vertical do vento e do escalar cujo fluxo se deseja medir. Segundo Salesky et al. (2012), as medições de fluxo pelo MCT podem possuir contribuições de vários tipos de erros, incluindo erros sistemáticos, erros devido à não estacionariedade das séries temporais, e erros aleatórios. O erro aleatório é definido como o erro devido a um período médio insuficiente para que a média no tempo convirja para a média probabilística. Esse tipo de erro pode ser uma fonte significativa de erro para os estudos de turbulência na camada limite atmosférica para escalas temporais pequenas.

Considerando somente o erro aleatório, Salesky et al. (2012) desenvolveram um método de filtragem para estimar o erro aleatório de momentos turbulentos de qualquer ordem através da decomposição espacial local dos fluxos turbulentos baseada em propriedades de filtros espaciais. Esse método, a priori, não precisa de uma estimativa da escala integral, a qual pode ser altamente sensível para o método utilizado e produzir incertezas na estimativa do erro. O método desenvolvido é baseado diretamente em calcular a variância do erro do fluxo local como uma função da escala $\Delta$ e extrapolar para estimar a variância do erro para o período $T$.

Levando em consideração essas questões, este trabalho tem como objetivo aplicar o método de filtragem de Salesky et al. (2012), usando um filtro temporal, para estimar o erro do fluxo de calor sensível a partir de dados obtidos em Tijucas do Sul - PR e em Missal - PR. 


\section{FORMULAÇÃO DO MÉTODO}

Sendo $f$ um processo estocástico com média probabilística $\langle f\rangle$ e variância $\left\langle f^{\prime 2}\right\rangle=$ $\left\langle[f-\langle f\rangle]^{2}\right\rangle$, Lumley e Panofksy (1964) definem o erro aleatório em função da variância do erro para um período médio $\mathrm{T}$ como

$$
\sigma_{\bar{f}}^{2}(T)=\left\langle\left[\frac{1}{T} \int_{t_{0}}^{t_{0}+T} f(t) d t-\langle f\rangle\right]^{2}\right\rangle=\left\langle[\bar{f}-\langle f\rangle]^{2}\right\rangle .
$$

Supondo que $f$ é estacionário, que a escala integral existe, e considerando as propriedades simétricas das funções de autocorrelação, Lumley e Panofksy (1964) encontraram que a variância do erro pode ser expressa por

$$
\sigma_{\bar{f}}^{2}(T)=\frac{2 T_{f}\left\langle f^{\prime 2}\right\rangle}{T}
$$

onde $T_{f}$ é a escala integral temporal de $f$, que por definição é dada por

$$
T_{f} \equiv \int_{0}^{\infty} \rho(\tau) d \tau
$$

onde $\rho(\tau)$ é a função de autocorrelação de $f$.

Aplicando a equação (1) para um dado filtro temporal de largura $\Delta$, obtemos

$$
\sigma_{\tilde{f}}^{2}(\Delta)=\left\langle\left[\frac{1}{\Delta} \int_{t_{0}}^{t_{0}+\Delta} f(t) d t-\langle f\rangle\right]^{2}\right\rangle=\left\langle[\tilde{f}-\langle f\rangle]^{2}\right\rangle .
$$

Agora mostraremos como Lumley e Panofksy (1964) chegaram até a equação (2), porém para a escala $\Delta$. Realizando manipulações algébricas em (4), temos

$\sigma_{\tilde{f}}^{2}(\Delta)=\frac{1}{\Delta^{2}} \int_{t=0}^{\Delta} \int_{\tau=0}^{\Delta}\langle[f(t)-\langle f\rangle][f(\tau)-\langle f\rangle]\rangle d \tau d t=\frac{1}{\Delta^{2}} \int_{t=0}^{\Delta} \int_{\tau=0}^{\Delta}\left\langle f^{\prime 2}\right\rangle \rho(\tau-t) d \tau d t$.

Fazendo a seguinte mudança de variáveis $x=t$ e $y=\tau-t$, invertendo a ordem de integração e resolvendo a integral em $x$, chegamos à seguinte equação:

$$
\sigma_{\tilde{f}}^{2}(\Delta)=\frac{\left\langle f^{\prime 2}\right\rangle}{\Delta^{2}}\left(\int_{y=-\Delta}^{0} \int_{x=0}^{-y} \rho(y) d x d y+\int_{y=0}^{\Delta} \int_{x=0}^{\Delta-y} \rho(y) d x d y\right)=\frac{2\left\langle f^{\prime 2}\right\rangle}{\Delta} \int_{y=0}^{\Delta}\left(1-\frac{y}{\Delta}\right) \rho(y) d y .
$$

Segundo Tennekes e Lumley (1972) se o tempo de integração $\Delta$ é muito maior que a escala integral $T_{f}, y / \Delta \sim 0$ no intervalo de valores de $y$ onde $\rho(y) \neq 0$, então usando a definição da escala integral, equação (3), a variância do erro pode ser aproximada por

$$
\sigma_{\tilde{f}}^{2}(\Delta)=\frac{2 T_{f}\left\langle f^{\prime 2}\right\rangle}{\Delta}
$$

\section{DESENVOLVIMENTO DO MÉTODO}

Agora, desenvolveremos o método para estimar o erro aleatório do fluxo de calor sensível $f=w^{\prime} \theta^{\prime}$. Tirando a raiz quadrada e supondo ergodicidade podemos reescrever (4) e (7) usando a média temporal: 


$$
\begin{gathered}
\sigma_{\widetilde{w^{\prime} \theta^{\prime}}}(\Delta)=\sqrt{\overline{\left[\widetilde{w^{\prime} \theta^{\prime}}-\overline{w^{\prime} \theta^{\prime}}\right]^{2}}}, \\
\sigma_{\widetilde{w^{\prime} \theta^{\prime}}}(\Delta)=\sqrt{2 T_{f}}\left[\overline{\left(w^{\prime} \theta^{\prime}-\overline{w^{\prime} \theta^{\prime}}\right)^{2}}\right]^{1 / 2} \Delta^{-1 / 2}=C_{w \theta} \Delta^{-1 / 2},
\end{gathered}
$$

onde $C_{w \theta}$ é uma constante.

Para estimar o erro do fluxo de calor sensível, é necessário ajustar $\widetilde{w^{\top} \theta^{\prime}}$, calculado por (8), na forma da lei de potência dada por (9), onde $C_{w \theta}$ é o coeficiente a ser determinado pelo método dos mínimos quadrados. Salesky et al. (2012) verificaram que os limites para fazer o ajuste da lei de potência são $\Delta_{\min }=10 T_{f}$ e $\Delta_{\max }=T / 10$, sendo que a escala de integral será aproximada por $T_{f} \sim z / \bar{u}$ onde $\bar{u}$ é a velocidade horizontal média, $z$ é a altura de medição e $T$ é o período de análise, neste trabalho $T=30$ minutos. Finalmente, o erro aleatório é estimado avaliando o ajuste da lei de potência (9) para $\Delta=T$ e normalizando pelo valor médio do fluxo:

$$
\epsilon_{w \theta}=\frac{\sigma_{\overline{w^{\prime} \theta^{\prime}}}(T)}{\overline{w^{\prime} \theta^{\prime}}}
$$

\section{MATERIAIS E MÉTODOS}

Os dados analisados neste trabalho são provenientes de duas campanhas micrometeorológicas, uma realizada em uma grameira em Tijucas do Sul - Paraná no período de 17/02/2011 até 27/02/2011 que possui 102 arquivos de 30 minutos e outra realizada em uma pequena ilha localizada no lago do reservatório de Itaipu nas proximidades da cidade de Missal - Paraná de 13/12/2012 até 17/12/2012 que possui 196 arquivos de 30 minutos. A aquisição dos dados nas duas campanhas foi realizada a $20 \mathrm{~Hz}$. Os dados necessários para calcular o fluxo de calor sensível (velocidade do vento e temperatura) foram obtidos a partir de um anemômetro sônico tridimensional CSAT3 e de um termopar de fio fino FW3, posicionado no centro dos transdutores do CSAT3, ambos da Campbell Scientific. Antes de aplicar o método de filtragem foi realizado o controle de qualidade nos dados, a rotação de coordenadas e por fim o cálculo das flutuações de acordo com a decomposição de Reynolds.

\section{RESULTADOS}

As Figuras 1 e 2 apresentam o desvio padrão do fluxo de calor sensível calculado pelos dados (linha preta) e ajustado aos dados por $\sigma_{\widetilde{w^{\prime} \theta^{\prime}}}(\Delta)=C_{w \theta} \Delta^{-1 / 2}$ no intervalo de $\Delta_{\min }=$ $10 T_{f}$ e $\Delta_{\max }=T / 10$ (linha pontilhada) respectivamente para Tijucas do Sul e Missal. Pela equação (10), temos que o desvio padrão do fluxo de calor sensível extrapolado para o período $T$ (ponto preto), normalizado pelo fluxo médio resulta no erro $\epsilon_{w \theta}$. Para a Figura 1 $1^{\mathrm{a}}$, temos que $\epsilon_{w \theta} \sim 6 \%$, para Figura $1 \mathrm{~b} \epsilon_{w \theta} \sim 7 \%$ e para as Figuras $2 \mathrm{a}$ e $2 \mathrm{~b} \epsilon_{w \theta} \sim 8 \%$. 

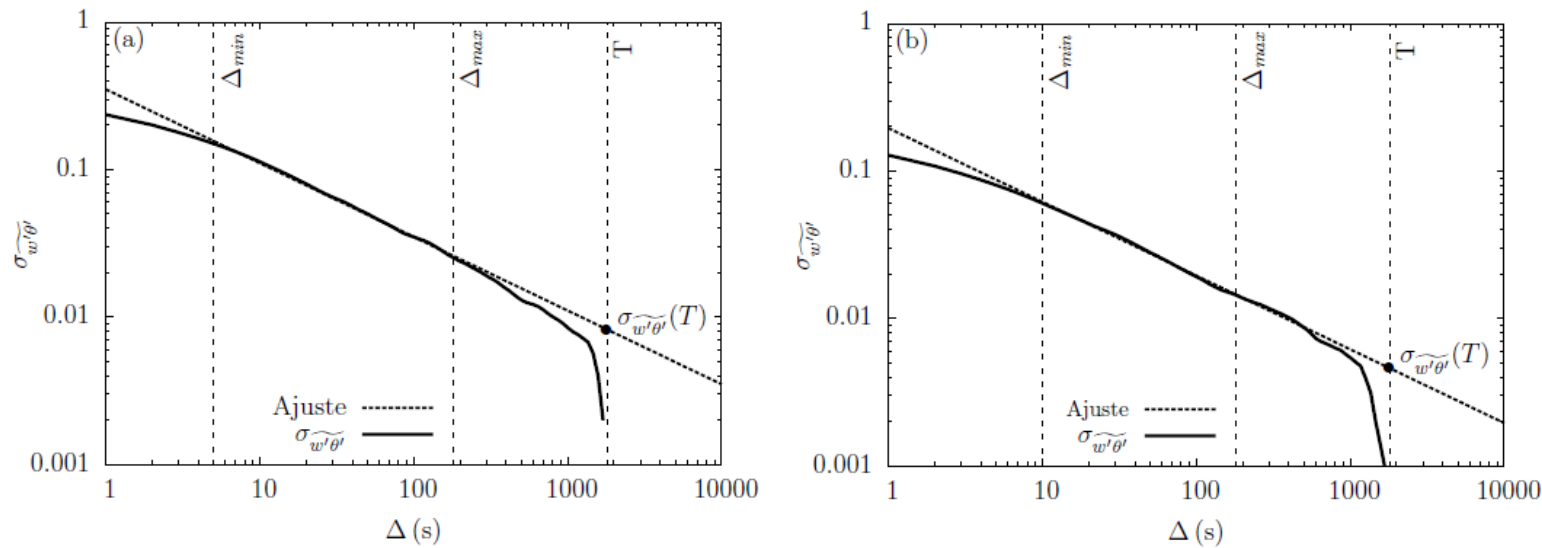

Figura 1. Desvio padrão do fluxo em Tijucas do Sul. (a) 19/02/2011 - 16:00. (b) 21/02/2011 - 12:10.
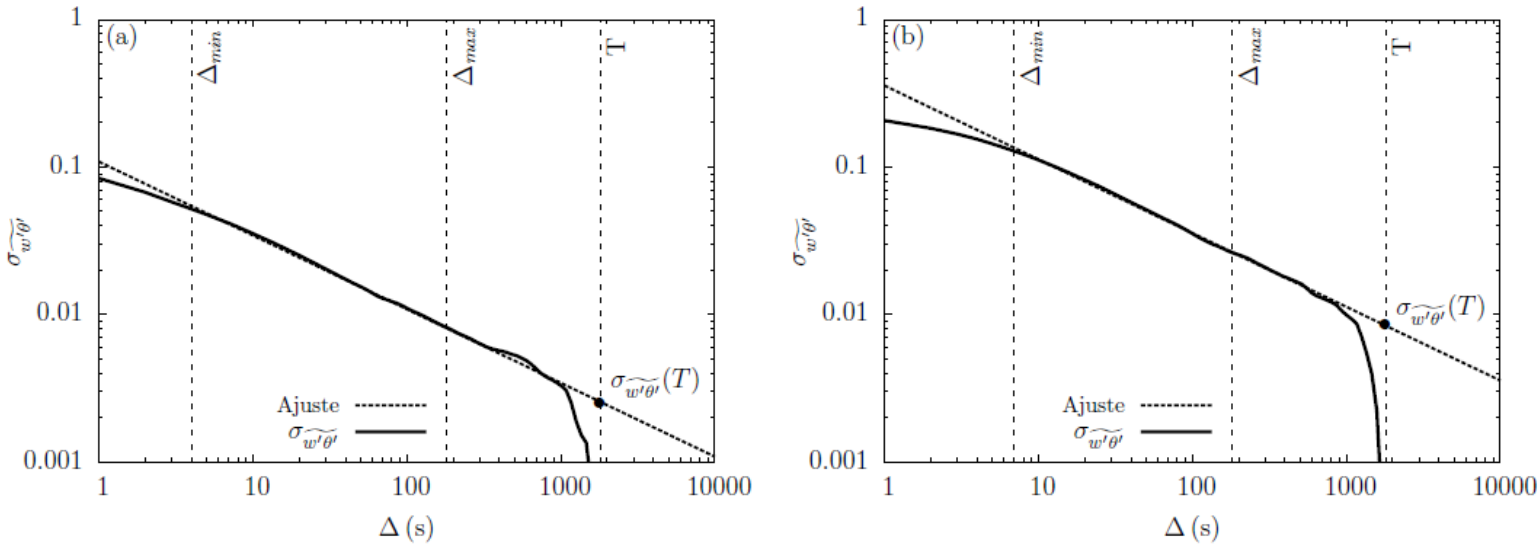

Figura 2. Desvio padrão do fluxo em Missal (a) 16/12/2012 - 10:00. (b) 16/02/2012 - 16:00.

Analisando os gráficos gerados após o método de filtragem, verificou-se que poucos deles decaem segundo a lei de potência $-1 / 2$. Para analisar qual a real lei de potência fizemos o seguinte ajuste $\sigma_{\widetilde{w^{\prime} \theta^{\prime}}}(\Delta)=C_{w \theta} \Delta^{a}$. A Figura 3 mostra um histograma com o resultado encontrado para o ajuste de $a$. Analisando a figura é possível verificar que, para ambas as situações, a maioria dos casos está no intervalo de $-0.3 \mathrm{a}-0.5$.
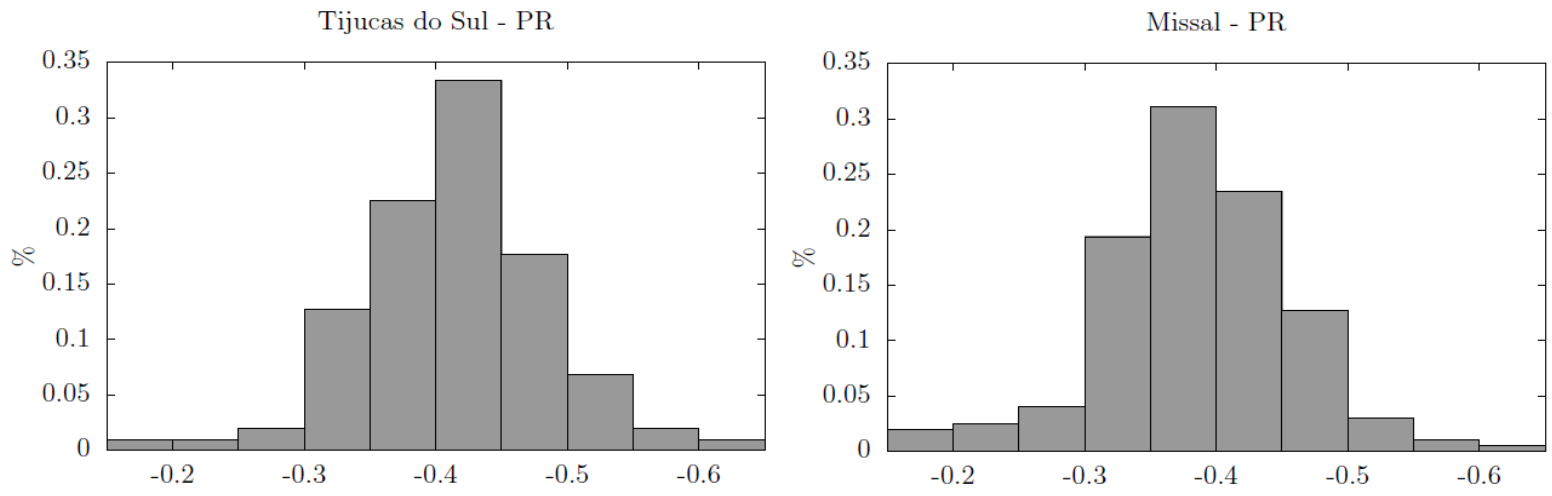

Figura 3. Histograma com os valores de $a$, resultantes do ajuste de $\sigma_{\widetilde{w^{\prime} \theta^{\prime}}}(\Delta)=C_{w \theta} \Delta^{a}$. 


\section{CONCLUSÃO}

Neste trabalho, aplicamos o método proposto por Salesky et al. (2012) para estimar o erro aleatório do fluxo de calor sensível, porém ao invés de usar um filtro espectral, usou-se um filtro temporal. Pelos resultados apresentados nas Figuras 1 e 2, verificamos que o filtro temporal também é aplicável a este método. $\mathrm{O}$ valor encontrado para o erro do fluxo de calor sensível foi aproximadamente de 7\%.

Conforme mostram os histogramas da Figura 3, a maioria dos casos segue a lei de potência no intervalo de -0.3 a -0.5 , o que difere da previsão teórica, equação (9). Salesky et al. (2012) comentam que o decaimento previsto pela teoria são casos ideais e que na prática se ajustarmos $\sigma_{\widetilde{w^{\prime} \theta^{\prime}}}(\Delta)=C_{w \theta} \Delta^{a}$, nem sempre se obtém $a=-1 / 2$. Eles acreditam que estas diferenças são devido a não-estacionariedade nas séries temporais.

\section{AGRADECIMENTOS}

Parte deste trabalho foi realizada com recursos do projeto FUNPAR 2882 financiado pela Companhia Hidro Elétrica do São Francisco (CHESF), da Chamada ANEEL 009/2008, Projeto Estratégico "Monitoramento das Emissões de Gases de Efeito Estufa em Reservatórios de Usinas Hidrelétricas", do Fundo Setorial de Energia Elétrica.

\section{REFERÊNCIAS}

LUMLEY, J. L.; PANOFKSY, H. A. The structure of atmospheric turbulence. New York: Interscience, 1964.

SALESKY, S. T.; CHAMECKI, M.; DIAS, N. L. Estimating the random error in eddycovariance based fluxes and other turbulence statistics: the filtering method.

Boundary-Layer Meteorology, v. 144, p. 113-135, 2012.

TENNEKES, H.; LUMLEY, J. L. A first course in turbulence. Cambridge, Massachusetts: The MIT Press, 1972 